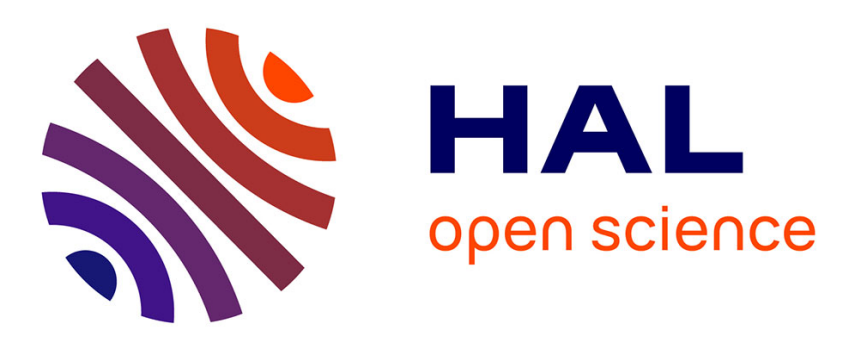

\title{
An AES study of damage induced by inert gas ions at SiO2 surfaces : influence of ion mass and energy
}

\author{
M. Khellafi, B. Lang
}

\section{To cite this version:}

M. Khellafi, B. Lang. An AES study of damage induced by inert gas ions at $\mathrm{SiO} 2$ surfaces: influence of ion mass and energy. Revue de Physique Appliquée, 1990, 25 (4), pp.389-394. 10.1051/rphysap:01990002504038900 . jpa-00246197

\section{HAL Id: jpa-00246197 https://hal.science/jpa-00246197}

Submitted on 1 Jan 1990

HAL is a multi-disciplinary open access archive for the deposit and dissemination of scientific research documents, whether they are published or not. The documents may come from teaching and research institutions in France or abroad, or from public or private research centers.
L'archive ouverte pluridisciplinaire HAL, est destinée au dépôt et à la diffusion de documents scientifiques de niveau recherche, publiés ou non, émanant des établissements d'enseignement et de recherche français ou étrangers, des laboratoires publics ou privés. 


\title{
An AES study of damage induced by inert gas ions at $\mathrm{SiO}_{2}$ surfaces : influence of ion mass and energy
}

\author{
M. Khellafi and B. Lang $(*)$ \\ Groupe «Surfaces-Interfaces», Institut de Physique et Chimie des Matériaux, Unité Mixte CNRS-ULP- \\ EHICS, 4 rue Blaise Pascal, F-67000 Strasbourg, France
}

(Reçu le 5 novembre 1989, révisé le 22 décembre 1989, accepté le 5 janvier 1990)

\begin{abstract}
Résumé. - L'endommagement de surfaces de $\mathrm{SiO}_{2}$ bombardées avec des ions $\mathrm{Ar}^{+}, \mathrm{Ne}^{+}$et $\mathrm{He}^{+}$d'énergie 70$500 \mathrm{eV}$ est étudié par spectroscopie Auger dans des conditions d'analyse non destructives. La couche perturbée est déficitaire en oxygène et contient de nouveaux états de liaison du silicium, caractérisés par les pics $\mathrm{SiO}_{x}(82 \mathrm{eV})$ et $\mathrm{Si}(89 \mathrm{eV})$. Les modifications observées en fonction de la masse et de l'énergie des ions renseignent sur le mécanisme de l'interaction ion-surface. A énergie moyenne $(500 \mathrm{eV})$, le changement de composition est compatible avec un modèle de pulvérisation par collisions élastiques. A basse énergie (80$250 \mathrm{eV}$ ), l'interaction de $\mathrm{He}^{+}$est totalement différente de celle de $\mathrm{Ar}^{+}$et $\mathrm{Ne}^{+}$, ce qui suggère l'entrée en jeu de processus inélastiques du type neutralisation Auger.
\end{abstract}

\begin{abstract}
The altered layer produced by sputtering $\mathrm{SiO}_{2}$ with $\mathrm{Ar}^{+}, \mathrm{Ne}^{+}$and $\mathrm{He}^{+}$ions of $70-500 \mathrm{eV}$ energy is studied by AES in conditions avoiding electron beam damage. The understoechiometric layer is characterized by an attenuation of the O KVV Auger peak and by the appearance of additional $\mathrm{SiO}_{x}(82 \mathrm{eV})$ and $\mathrm{Si}(89 \mathrm{eV})$ peaks in the Si LVV spectrum. The dependence of this damage upon ion mass and ion energy gives information on the mechanism of ion-surface interaction. At medium energy $(500 \mathrm{eV})$, the change in surface composition is compatible with a collisional sputtering model. At lower energies $(80-250 \mathrm{eV}), \mathrm{He}^{+}$interacts quite differently from $\mathrm{Ar}^{+}$and $\mathrm{Ne}^{+}$, suggesting the onset of inelastic processes like Auger neutralization.
\end{abstract}

\section{Introduction.}

The present work is part of a systematic study of radiation damage at oxide surfaces, induced by inert gas ions with energies typical of sputter-cleaning and by electrons with energies typical of AES, i.e. energies which the particles may possess in a cold plasma environment. The Auger probe itself may have a damaging effect, so that one has to discriminate between true ion beam damage and additional damage due to the electrons.

Once the operating conditions for least-damaging analysis are determined (fluence : $<10^{18} \mathrm{e} / \mathrm{cm}^{2}[1]$, energy : $2500 \mathrm{eV}$ [2]), one may isolate the changes due to the sole ion bombardment and investigate their dependence upon ion mass and ion energy. This is what we attempt here.

$\left(^{*}\right)$ To whom correspondence should be sent.

\section{Experimental.}

The Auger analysis is performed with a single-pass CMA in an ultra-high vacuum chamber, which is separated from the ion pump by a gatevalve.

The ion gun is of the «ion gauge " type. It is operated in the static mode : $10^{-4}$ torr inert gas, ion pump isolated, Ti sublimation pump on to keep the residual pressure of active gas low. Typical working conditions are : 70-500 eV ion energy, normal incidence, $1-10 \mu \mathrm{A} / \mathrm{cm}^{2}$ ion current density. The fluence used corresponds to damage saturation, respectively : $4 \times 10^{15}\left(\mathrm{Ar}^{+}\right), \quad 2 \times 10^{16}\left(\mathrm{Ne}^{+}\right)$and $1-2 \times 10^{17}\left(\mathrm{He}^{+}\right)$ions $/ \mathrm{cm}^{2}$ [1]. After completion of the bombardment, the inert gas is pumped away via an auxiliary UHV diffusion pump before reopening the ion pump. In this way, the pressure is back in the low $10^{-9}$ torr range two minutes after the end of the bombardment. Rapid cycling between bombardment at $10^{-4}$ torr and analysis at $10^{-9}$ torr is thus allowed without overloading the ion pump with inert gas. 
Typical operating conditions for Auger work are : primary energy $2500 \mathrm{eV}$, primary current $10^{-7} \mathrm{~A}$, modulation $2 V_{\mathrm{p}-\mathrm{p}}$, scan speed $25 \mathrm{eV} / \mathrm{min}$, time constant $0.3 \mathrm{~s}$. The duration of a scan corresponds to a fluence of less than $10^{18} \mathrm{e} / \mathrm{cm}^{2}$. The nominal beam diameter is $0.1 \mathrm{~mm}$; the current is measured with the sample holder biased $+90 \mathrm{~V}$ relative to ground.

The $\mathrm{SiO}_{2}$ sample is a $3500 \AA$ thick dry oxide grown on $\mathrm{Si}$ (of the same type as used in Refs. [1, 2]). It is mounted on a Ta-covered NB block heated by a $\mathrm{W}$ resistor. The reference state (« undamaged» surface) is obtained after 5 min annealing at $700 \mathrm{C}$ in the UHV ambient [1]. Notice that the sample « history " (numerous sputtering + annealing cycles) is markedly different from that of a sample only sputtered and never heated. While this may affect macroscopic properties, we have no evidence that the surface composition would be different.

\section{Results.}

AES gives evidence for ion-induced damage at $\mathrm{SiO}_{2}$ surfaces in the form of compositional changes (peak height, peak shape) and electrical changes (peak displacement due to charging) $[1,2]$. Here, we restrict ourselves to the former type of changes. The modifications are evaluated with respect to the reference spectrum, which is regenerated by annealing before each new ion bombardment. All results given below relate to the freshly ion-bombarded surface with minimum electron beam exposure.

Changes typical of ion bombardment consist in a slight attenuation of the $\mathrm{O} \mathrm{KVV}$ peak $(502 \mathrm{eV})$ and large changes in the Si LVV spectrum (Fig. 1) whereby the $\mathrm{SiO}_{2}$ peak $(77 \mathrm{eV})$ loses intensity at the benefit of new features appearing on its high-energy wing near 82 and $89 \mathrm{eV}$. The $89 \mathrm{eV}$ peak is called " free $\mathrm{Si}$ » or " elemental $\mathrm{Si}$ » peak ; the intermediate $82 \mathrm{eV}$ peak is called « $\mathrm{SiO}_{x}$ peak ». Further exposure to the electron beam (Fig. 1c) would lead to the decay of the $\mathrm{SiO}_{x}$ peak and the growth of the $\mathrm{Si}$ peak.

3.1 IONS OF SEVERAL HUNDREDS OF eV. - The $\mathrm{O} K V V$ peak suffers an attenuation, which depends on the nature of the projectile. For $500 \mathrm{eV}$ ions, we find mean attenuations rising with decreasing mass : $7 \%(\mathrm{Ar}), 11 \%(\mathrm{Ne}), 17 \%(\mathrm{He})$.

Turning to the Si LVV spectrum, we investigate the behavior of the damage features $\left(\mathrm{SiO}_{x}\right.$ and $\mathrm{Si}$ peaks) as a function of the main parameters of the ion bombardment (fluence, energy, mass). We note (Fig. 2) that the $\mathrm{SiO}_{x}$ peak appears for lower fluences than the Si peak. Taking the example of $\mathrm{Ne}^{+}$ions where the saturation fluence is $2 \times 10^{16}$ ions $/ \mathrm{cm}^{2}$ [1], the $\mathrm{SiO}_{x}$ peak is already detected (Fig. 2) for $5 \times 10^{14}$ ions $/ \mathrm{cm}^{2}$, while the Si peak appears around $3 \times 10^{15}$ ions $/ \mathrm{cm}^{2}$, i.e. respectively $2.5 \%$ and $15 \%$

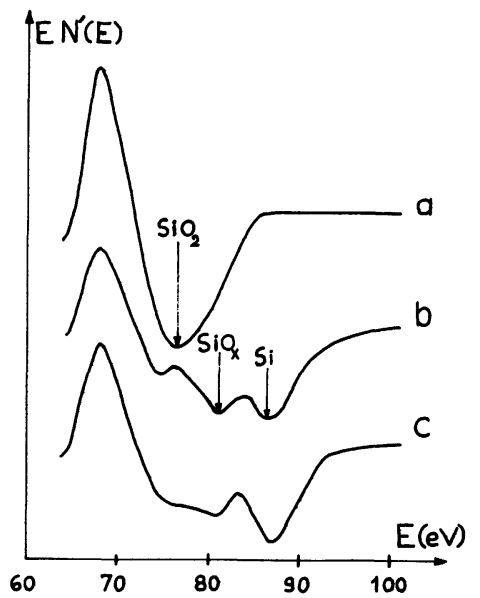

Fig. 1. - Si LVV Auger spectra of $\mathrm{SiO}_{2}$ surfaces : (a) undamaged ; (b) freshly bombarded with $250 \mathrm{eV} \mathrm{Ne}^{+}$; (c) as (b), but with additional $15 \mathrm{~min}$ exposure to the electron beam. Mod. $2 V_{\mathrm{p}-\mathrm{p}}, 2500 \mathrm{eV}$ electrons.

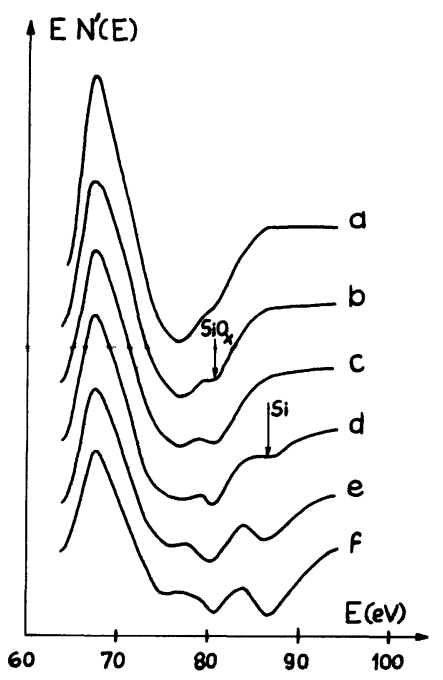

Fig. 2. - Modification of Si LVV Auger spectrum of $\mathrm{SiO}_{2}$ as a function of ion fluence $\left(\mathrm{Ne}^{+}, 250 \mathrm{eV}\right)$ : (a) undamaged ; (b) $5 \times 10^{14}$; (c) $1.5 \times 10^{15}$; (d) $3 \times 10^{15}$; (e) $7 \times 10^{15}$; (f) $1,5 \times 10^{16}$ ions $/ \mathrm{cm}^{2}$. Mod. $1 V_{\mathrm{p}-\mathrm{p}} ; 2500 \mathrm{eV}$ electrons.

of the saturation fluence. In addition, when decreasing the ion energy (Fig. 3), the $\mathrm{SiO}_{x}$ peak disappears later than the $\mathrm{Si}$ peak. Thus, the $\mathrm{SiO}_{x}$ is detectable at lower damage densities than the Si peak. Note that, even on undamaged $\mathrm{SiO}_{2}$, a small $\mathrm{SiO}_{x}$ type inflection may be detected with improved resolution (modulation $1 V_{\mathrm{p} . \mathrm{p}}$ or less), which is the case in figure 2 and reference [3].

Further tendencies, like the increase of the damage saturation fluence and of the Si peak height with decreasing ion mass, have already been reported [1] and may be understood by a decreasing sputter rate and an increasing ion range. 


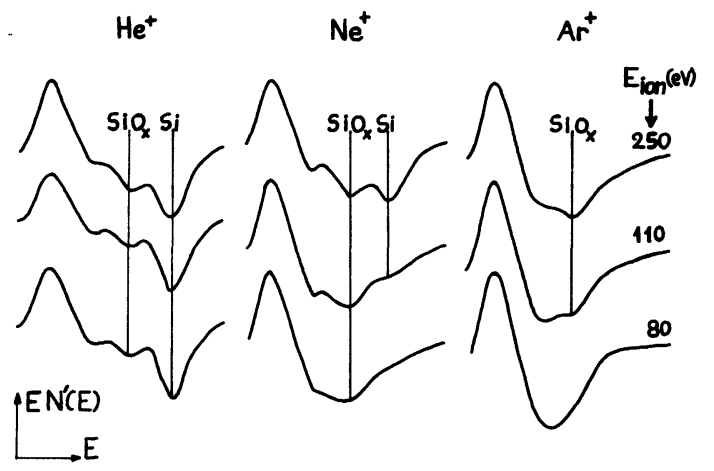

Fig. 3. - Si LVV Auger spectra of ion bombarded $\mathrm{SiO}_{2}$ : evolution of $\mathrm{SiO}_{x}$ and $\mathrm{Si}$ peaks as a function of ion mass and ion energy (raw spectra : heights unnormalized to reference surface). Mo. $2 V_{\mathrm{p}-\mathrm{p}} ; 2500 \mathrm{eV}$ electrons.

Finally, note that, whereas the Auger peak of implanted $\operatorname{Ar}(216 \mathrm{eV})$ is easily measured in ionbombarded $\mathrm{Si}$, it escapes detection in ion-bombarded $\mathrm{SiO}_{2}$ (probably due to a larger penetration).

\subsection{IONS IN THE LOW-ENERGY RANGE AROUND} $100 \mathrm{eV}$. - Over the energy range investigated here and even beyond (up to $1.5 \mathrm{keV}$ ) $\mathrm{Ar}^{+}$ions differ from $\mathrm{Ne}^{+}$and $\mathrm{He}^{+}$ions by the absence of the free $\mathrm{Si}$ peak. The only damage feature induced by $\mathrm{Ar}^{+}$ions is the $\mathrm{SiO}_{x}$ peak which has a threshold at 100 $( \pm 10 \mathrm{eV})$. In the case of $\mathrm{Ne}^{+}$, the $\mathrm{SiO}_{x}$ peak has its threshold at $75( \pm 5 \mathrm{eV})$; for $\mathrm{He}^{+}$, the $\mathrm{SiO}_{x}$ peak is still large at $80 \mathrm{eV}$, which is the minimum energy we could achieve with this gas (Fig. 3).

Consider next the behaviour of the free $\mathrm{Si}$ yield as a function of ion energy, for the two species giving rise to a measurable free $\mathrm{Si}$ signal $\left(\mathrm{Ne}^{+}, \mathrm{He}^{+}\right)$. The free $\mathrm{Si}$ yield, or dissociation yield, is defined as the ratio of the free Si peak height to the height of the $\mathrm{SiO}_{2}$ peak of the reference surface [2]. There is a striking difference between $\mathrm{Ne}^{+}$and $\mathrm{He}^{+}$(Fig. 4). For $\mathrm{Ne}^{+}$, the yield decreases and vanishes around $80 \mathrm{eV}$. For $\mathrm{He}^{+}$, the yield starts rising below $250 \mathrm{eV}$ and increases steadily up to $80 \mathrm{eV}$ (limit of our measurements). A maximum would probably be attained for a few tens of $\mathrm{eV}$.

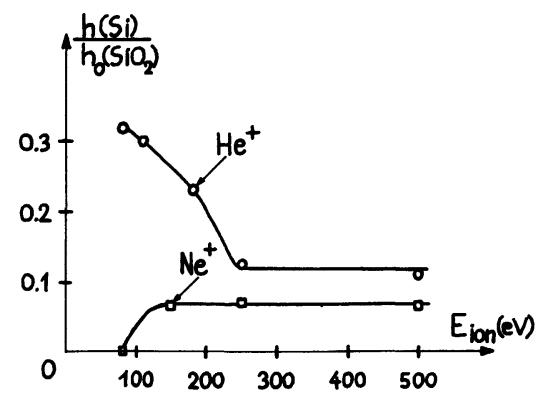

Fig. 4. - Variation of free Si yield as a function of ion energy for $\mathrm{Ne}^{+}$and $\mathrm{He}^{+}$.

\section{Discussion.}

The discussion is organized as follows. Firstly, we discuss the change in bonding representative of ioninduced damage, as compared to damage caused by the electron beam only or else by the combination of ion and electron bombardment. Next, we propose a semiquantitative evaluation of the composition of the altered layer and compare to calculations based on collisional sputtering. Then, we discuss the dependence of damage upon ion energy and ion mass, which raises questions about the mechanism of the interaction. Finally, we briefly comment upon the $\mathrm{SiO}_{2}$-type sensitivity of the damage.

4.1 BONDING STATES IN THE ALTERED LAYER. Ion-induced damage is characterized by two features : the $\mathrm{SiO}_{x}$ and the $\mathrm{Si}$ peaks, which coexist in various proportions (according to ion mass and ion energy). The $\mathrm{SiO}_{x}$ peak is attributed to a suboxide component, which is metastable and behaves similarly to vacuum-deposited $\mathrm{SiO}$ films [4]. For more details, see reference [1]. The Si peak is attributed to small clusters of elemental Si $[1,5,6]$. This picture of the ion-bombarded surface is in agreement with findings on other oxides [7], which show the coexistence of the initial oxide, the suboxide and the metal.

By contrast, electron bombardment alone does not generate the $\mathrm{SiO}_{x}$ feature $[1,2,8]$. Thus, although its detailed nature is not known, the $\mathrm{SiO}_{x}$ configuration appears connected with collisional sputtering and should belong to the class of vacancy-related centers. The Si component is generated as well by the lighter inert gas ions as by electrons $[1,2,8]$. Therefore, a participation of electronic sputtering (bond-breaking) is not excluded.

When electron bombardment follows ion bombardment (Fig. 1c), the dominant damage feature becomes the free Si peak (due to the metastability of the $\mathrm{SiO}_{x}$ component) $[1,2]$. The $\mathrm{SiO}_{x}$ disproportionates into $\mathrm{Si}$ and $\mathrm{SiO}_{2}[2]$; this electron-induced rearrangement occurs at constant $O$ content, i.e. the $\mathrm{O}$ deficiency is fixed by the ion bombardment. Turned differently, the ion-bombarded surface is stabilized against further $O$ loss due to the electron beam.

The case of simultaneous ion and electron bombardment (which is of interest in Auger sputter profiling and in plasma treatments) is not feasible in our apparatus. Nevertheless, one can extrapolate and expect the resulting surface composition to depend on the ratio of electron to ion current density (i.e. of dissociation to sputter rate) : when this ratio increases, the Si component should increase at the expense of the $\mathrm{SiO}_{x}$ component. This tendency is implicitely contained in reference [9], figure 7.

Considering the advantage of photoemission in terms of damage and ease of interpretation of the 
fine structure, it would be interesting to investigate the Si $2 p$ bonding states by surface-sensitive photoemission.

4.2 Composition of THE Altered layer. - Auger peak intensities can be used to obtain semiquantitative information about the composition of the ion-damaged surface. From the measured attenuation of the $\mathrm{O}$ Auger peak, one can evaluate the $\mathrm{O}$ loss.

Suppose that the $O$ deficit is concentrated in a thin surface layer of thickness $\lambda / 2, \lambda$ being the inelastic mean free path of the $O$ Auger electrons in $\mathrm{SiO}_{2}$ (about 10-15 $\AA$ ) and that this thickness is independent of the ion penetration. This assumption is based on following arguments : a) the shallow depth of origin of sputtered particles [10, 11] ; b) the localization of the dissociation in the first few atomic layers, thus escaping detection by the less surface-sensitive XPS probe [2] ; c) the insensitivity of the dissociation yield to ion energy above $300 \mathrm{eV}$ (Fig. 4).

From the exponential variation of Auger intensity as a function of depth $D: I / I_{0}=\exp ^{-}(D / \lambda)$, we calculate that $40 \%$ of the Auger intensity originates from the top $\lambda / 2$ thick layer. The Auger intensity is taken as proportional to the peak-to-peak height of the derivative peak. Calling $h_{0}(\mathrm{O})$ the $\mathrm{O}$ peak height of the reference surface, $h_{\mathrm{d}}(\mathrm{O})$ the $\mathrm{O}$ peak height of the damaged surface and $x$ the atomic ratio $\mathrm{O} / \mathrm{Si}$, we may write the proportionality of the number of $O$ atoms in a slab of thickness $\lambda / 2$ to the Auger intensity :

$$
\frac{x}{2}=\frac{h_{\mathrm{d}}(\mathrm{O})-h_{0}(\mathrm{O}) \times 0.6}{h_{0}(\mathrm{O})-h_{0}(\mathrm{O}) \times 0.6}
$$

$0.6 h_{0}(\mathrm{O})$ representing the contribution from the undamaged substrate below the altered layer. Introducing the attenuation of the Auger intensity: $A=1-h_{\mathrm{d}} / h_{0}$, we get : $x=2(1-A / 0.4)$; the coefficient 0.4 is directly related to the assumed depth of the altered layer. The values of the calculated atomic ratios are given in table $\mathrm{I}$.

Table I. - Composition of damaged surface layer from AES.

\begin{tabular}{cccc}
\hline ion & $A(\%)$ & $x$ & Composition \\
\hline $\mathrm{Ar}^{+}$ & 7 & 1.65 & $\mathrm{SiO}_{1.65}$ \\
$\mathrm{Ne}^{+}$ & 11 & 1.45 & $\mathrm{SiO}_{1.45}$ \\
$\mathrm{He}^{+}$ & 17 & 1.15 & $\mathrm{SiO}_{1.15}$ \\
\hline
\end{tabular}

( $A=$ attenuation of $\mathrm{O}$ Auger peak ; $x=$ atomic fraction $\mathrm{O} / \mathrm{Si}$ ).
It is worth checking whether the composition deduced from AES is compatible with that predicted by the preferential sputtering model.

We use the formula applicable to the oxide of a metal $\mathbf{M}[13]$ :

$$
\left(X_{\mathrm{O}} / X_{\mathrm{M}}\right)_{\mathrm{s}}=\left(X_{\mathrm{O}} / X_{\mathrm{M}}\right)_{\mathrm{b}} \times\left(Y_{\mathrm{M}} / Y_{\mathrm{O}}\right)
$$

which relates the surface (s) and bulk (b) atomic fractions $X$ of the constituents to their sputtering yields $Y$.

The sputtering yields may be evaluated from [14] :

$$
Y_{M} / Y_{O}=\alpha_{M} / \alpha_{O} \times \gamma_{M} / \gamma_{O} \times U_{0}(\mathrm{O}) / U_{0}(\mathrm{M})
$$

where $\alpha$ depends on the ratio of ion mass to target atom mass and may be found in reference [9], figure $3, \gamma$ is the usual kinematical factor and $U_{0}$ the surface binding energy. The $U_{0}$ 's being less well-known quantities, we replace their ratio by a constant $K$.

Putting $\mathrm{M}=\mathrm{Si}$ and $\left(X_{\mathrm{O}} / X_{\mathrm{Si}}\right)_{\mathrm{b}}=2$, the calculated atomic fraction in the altered layer is respectively : $1.36 \mathrm{~K}\left(\mathrm{Ar}^{+}\right), 1.29 \mathrm{~K}\left(\mathrm{Ne}^{+}\right)$and $0.80 \mathrm{~K}\left(\mathrm{He}^{+}\right)$, which scales roughly with the experimental values (Tab. I), respectively : $1.65,1.45$ and 1.15 .

Fitting calculated to experimental values, $K$ can be estimated as : $0.6\left(\mathrm{Ar}^{+}\right), \quad 0.56\left(\mathrm{Ne}^{+}\right)$and $0.71\left(\mathrm{He}^{+}\right)$, so that the mean value is close to : $K=U_{0}(\mathrm{O}) / U_{0}(\mathrm{Si})=2 / 3$. In a simple interpretation the surface binding energy is proportional to the number of bonds. This naive argument seems to work in the present case. Indeed, if one neglects low-binding energy species like non-bridging $O$, the surface undergoing sputtering would mainly contain trivalent $\mathrm{Si}$ and divalent (bridging) $\mathrm{O}$.

To sum up, in the medium ion energy range, the composition of the surface layer, deduced from AES, agrees reasonably well with that predicted by collisional sputtering.

4.3 Mechanisms. - The question of the mechanisms involved is raised by observations made as a function of ion mass in the low-energy range.

Consider first the behavior of the $\mathrm{SiO}_{x}$ peak. Damage thresholds occur in the region $75\left(\mathrm{Ne}^{+}\right)$to $100 \mathrm{eV}\left(\mathrm{Ar}^{+}\right)$, which seems reasonable if the sputtering yield becomes negligible. Note that several authors, investigating the $\mathrm{Ar}^{+} / \mathrm{SiO}_{2}$ system, measure sputtering yields down to $40 \mathrm{eV}$ [15]. Hence, there should be an energy range, between $40-100 \mathrm{eV}$, within which $\mathrm{SiO}_{2}$ may be sputtered by $\mathrm{Ar}^{+}$ions without suffering any damage (at least as detected by AES).

Turning to the Si peak, we notice a fairly constant free $\mathrm{Si}$ yield in the medium energy range for both $\mathrm{Ne}^{+}$and $\mathrm{He}^{+}$. However, at lower energies, there is a drastic difference between the two projectiles. In the former case, the yield decreases to zero as the 
sputtering threshold is approached. In the latter case, the yield increases continuously when the energy is lowered in the range $80-250 \mathrm{eV}$ (Fig. 4). This may be attributed to the intervention of a new process involving, either a different type of elastic collision, or inelastic collisions leading to electronic excitation of the $\mathrm{Si}-\mathrm{O}$ bond followed by bond rupture. Both points of view may be found in the literature [16, 17], when trying to interpret the change of composition of oxides as a function of ion mass and energy.

Consider first elastic collisions. Two processes (in the case of normal incidence) may cause sputtering $[18,19]$ :

- type I : ejection by the projectile after its reflection on a subsurface atom;

- type II : ejection by one of the target atoms set in motion during the microcascade generated by the projectile. Several target atoms must be involved in order to reorientate the momentum outwards.

For $\mathrm{Ne}^{+}$(mass comparable to target atoms), only process II needs to be considered; it becomes inefficient when the number of displaced atoms becomes too small.

For $\mathrm{He}^{+}$(much lighter than target atoms), both processes are feasible. The change of slope observed near 200-250 eV (Fig. 4) may be assigned to a transition between type I and type II, the former predominating at lower energies. However, it is not clear why backscattered $\mathrm{He}$ would sputter $\mathrm{O}$ atoms much more efficiently than Si atoms, leading to the enhancement of the free Si component. Possibly, electronics effects play a part here by modifying the ratio of surface binding energies $U_{0}(\mathrm{O}) / U_{0}(\mathrm{Si})$.

Indeed, a different interpretation may be seeked, based on inelastic collisions involving ionization, electronic excitation [20]. The result may be, either bond rupture and desorption, or bond weakening, which means reduced surface binding energy, hence increased sputtering. In that regard, $\mathrm{O}$ atoms (electronegative species) would be more affected. In favor of this kind of explanation is the observation that the free Si component can be produced as well by ions as by electrons (see 4.1 ) or even by photons [21], i.e. by ionizing radiation.

Among the various electronic effects, consider in particular Auger neutralization (AN) of the low energy inert gas ion, a process thoroughly investigated by Hagstrum [22] in the case of metals and semiconductors. The general trends for the Auger neutralization yield and for the free Si dissociation yield are similar. Both increase when the ion becomes lighter, which is related to the increase in ionization energy. Moreover, both yields increase when the ion energy is lowered in the range between several hundreds of $\mathrm{eV}$ and a few tens of $\mathrm{eV}$.

Within the surface layer, AN represents a damaging event : the $\mathrm{Si}-\mathrm{O}$ bond goes through an excited state containing two holes in the valence band, which means weakening or rupture of the bond.

Outside the surface, AN shows up by electron emission. Using the appropriate terminology, $\mathrm{AN}$ is a source of potential emission, as opposed to kinetic emission which predominates at higher energies. An ion velocity of about $1 \times 10^{7} \mathrm{~cm} / \mathrm{s}$ is considered typical of the upper limit of the potential emission regime... For $\mathrm{He}^{+}$ions, this is precisely the velocity, corresponding to an energy of $250 \mathrm{eV}$, near which a change of slope in the upper curve of figure 4 occurs.

4.4 COMMENTS ON $\mathrm{SiO}_{2}$-TYPE SENSITIVITY OF THE DAMAGE. - The nature and the creation efficiency of bulk defects at higher energies (implantation range : tens to hundreds of $\mathrm{keV}$ ) are known to depend markedly on the precise nature of the target : crystalline or vitreous [24], wet or dry oxide [25], etc.

A similar influence is expected for surface damage induced by low energy particles; e.g. preliminary work on a thick wet oxide shows considerably less radiation damage than with the present thin dry oxide. Therefore, the behavior of a particular type of $\mathrm{SiO}_{2}$ sample should not be generalized without caution to other varieties (differing by their structure, processing, impurity content, etc.).

\section{References}

[1] LANG B., Appl. Surf. Sci. 37 (1989) 63.

[2] KHELlAFI M. and LANG B. (accepted for publication, Appl. Phys. A).

[3] LANG B. and Mosser A., J. Microsc. Spectrosc. Electron. 6 (1981) 131.

[4] Rochet F., Dufour G., Roulet H., Pelloie B., Perriere J., Fogarassy E., Slaoui A. and Froment M., Phys. Rev. B 37 (1988) 6468.

[5] Bermudez V. M. and Ritz V. H., Phys. Rev. B 20 (1979) 3446.
[6] JARDIN C., Journées d'Etudes sur les Surfaces Isolantes, Le Vide-Les Couches Minces, Suppl. to $n^{\circ} 243$ (1988) p. 95.

[7] LANG B., Interactions Plasmas Froids-Matériaux (Les Editions de Physique, Les Ulis) 1988, p. 283.

[8] Carrière B. and LANG B., Surf. Sci. 64 (1977) 209.

[9] Johannessen J. S., SPiCer W. E. and STRausSer Y. E., J. Appl. Phys. 47 (1976) 3028.

[10] Blaise G., and Coudray C., Utilisation de la 
Pulvérisation pour l'Analyse en Profondeur et le Traitement des Surfaces, Le Vide-Les Couches Minces, Suppl. to $\mathrm{n}^{\circ} 224$ (1984) p. 70.

[11] WitTMAACK K., Surf. Sci. 89 (1979) 668.

[12] HofmanN S. and Thomas J. H., J. Vac. Sci. Technol. B 1 (1983) 43.

[13] Malherbe J. B., Hofmann S. and Sanz J. M., Appl. Surf. Sci. 27 (1986) 355.

[14] Carter G., Nobes M. J. and Armour D. G., Vacuum 32 (1982) 509.

[15] Todorov S. S. and Fossum E. R., Appl. Phys. Lett. 52 (1988) 365, and references therein.

[16] Christie A. B., Sutherland J. and Walls J. M., Vacuum 31 (1981) 513.

[17] Taglauer E., Heiland W. and MacDonald R. J., Surf. Sci. 90 (1979) 661.

[18] Winters H. F. and Sigmund P., J. Appl. Phys. 45 (1974) 4760.
[19] Blaise G., Interactions Plasmas Froids-Matériaux (Les Editions de Physique, Les Ulis) 1988, p. 259.

[20] Kelly R., Surf. Sci. 90 (1979) 280.

[21] Fiori C. and Devine R. A. B., Induced Defects in Insulators, Ed. P. Mazzoldi (Les Editions de Physique, Les Ullis) 1984, p. 93.

[22] Hagstrum S., Inelastic Ion-Surface Collisions, Ed. N. H. Tolk (Academic Press) 1977, p. 1.

[23] Pivin J. C., Utilisation de la Pulvérisation pour l'Analyse en Profondeur et le Traitement des Surfaces, Le Vide-Les Couches Minces, Suppl. to $\mathrm{n}^{\circ} 224$ (1984) p. 189.

[24] Griscom D. L., The Physics of $\mathrm{SiO}_{2}$ and its Interfaces, Ed. S. T. Pantelides (Pergamon) 1978, p. 232.

[25] Devine R. A. B., J. Non Cryst. Solids, 107 (1988) 41. 